\title{
A rare cause of primary adrenal insufficiency due to a homozygous Arg188Cys mutation in the STAR gene
}

\author{
Lukas Burget1, Laura Audí Parera², Monica Fernandez-Cancio², Rolf Gräni', Christoph Henzen' and \\ Christa E Flück ${ }^{3}$
}

1Division of Endocrinology and Diabetes, Cantonal Hospital Lucerne, Lucerne, Switzerland, 2Pediatric Endocrinology Research Unit, Vall d’Hebron, Institut de Recerca, CIBERER, Instituto de Salud Carlos III, Barcelona, Spain, and 3Pediatric Endocrinology, Diabetology and Metabolism, Bern University Children's Hospital and Department of BioMedical Research, University of Bern, Bern, Switzerland

\author{
Correspondence \\ should be addressed \\ to L Burget \\ Email \\ lukas.burget@luks.ch
}

\section{Summary}

Steroidogenic acute regulatory protein (STAR) is a key protein for the intracellular transport of cholesterol to the mitochondrium in endocrine organs (e.g. adrenal gland, ovaries, testes) and essential for the synthesis of all steroid hormones. Several mutations have been described and the clinical phenotype varies strongly and may be grouped into classic lipoid congenital adrenal hyperplasia (LCAH), in which all steroidogenesis is disrupted, and non-classic LCAH, which resembles familial glucocorticoid deficiency (FGD), which affects predominantly adrenal functions. Classic LCAH is characterized by early and potentially life-threatening manifestation of primary adrenal insufficiency (PAI) with electrolyte disturbances and 46,XY disorder of sex development (DSD) in males as well as lack of pubertal development in both sexes. Non-classic LCAH manifests usually later in life with PAI. Nevertheless, life-long follow-up of gonadal function is warranted. We describe a 26 -year-old female patient who was diagnosed with PAI early in life without detailed diagnostic work-up. At the age of 14 months, she presented with hyperpigmentation, elevated ACTH and low cortisol levels. As her older brother was diagnosed with PAI two years earlier, she was put on hydrocortisone and fludrocortisone replacement therapy before an Addisonian crisis occurred. Upon review of her case in adulthood, consanguinity was noted in the family. Genetic analysis for PAI revealed a homozygous mutation in the STAR gene (c.562C>T, p.Arg188Cys) in both siblings. This mutation has been previously described in non-classic LCAH. This case illustrates that early onset, familial PAI is likely due to autosomal recessive genetic mutations in known genes causing PAI.

\section{Learning points:}

- In childhood-onset PAI, a genetic cause is most likely, especially in families with consanguinity.

- Adult patients with an etiologically unsolved PAI should be reviewed repeatedly and genetic work-up should be considered.

- Knowing the exact genetic diagnosis in PAI is essential for genetic counselling and may allow disease-specific treatment.

- Young men and women with NCLAH due to homozygous STAR Arg188Cys mutation should be investigated for their gonadal function as hypogonadism and infertility might occur during puberty or in early adulthood. 


\section{Background}

Primary adrenal insufficiency (PAI) comprises a large group of rare disorders with a broad spectrum of pathomechanisms and clinical phenotypes, e.g. syndromic and non-syndromic forms (1). In general, PAI is characterized by a lack or deficiency of production of adrenal steroids, specifically glucocorticoids, with consecutive activation of the CRH-ACTH axis and also, in case of concomitant mineralocorticoid deficiency, a negative feedback activation of the renin-angiotensin system. While in childhood genetic forms predominate, autoimmune PAI is the diagnosis most often found in adults. Overall, congenital adrenal hyperplasia $(\mathrm{CAH})$ due to 21-hydroxylase deficiency (CYP21A2 gene) is the most common form of genetic PAI (2). However, the etiologic spectrum comprises not only defects of steroid hormone biosynthesis, but also syndromes, adrenal dysgenesis, disorders characterized by ACTH resistance (also known as familial glucocorticoid deficiencies (FGD)), metabolic conditions with mitochondrial or peroxisomal defects, as well as disorders of cholesterol synthesis (3). Replacement therapy with glucocorticoids and mineralocorticoids, as needed, is essential, but remains often challenging with chronic overtreatment resulting in Cushings and undertreatment increasing the risk for a life-threatening Addison crisis (4).

- This case illustrates that work-up of an etiologically unsolved PAI in an adult patient might be complicated and only be solved by genetic analysis. Clinical signs and laboratory results are not precise enough and do overlap with other forms of PAI.

- To our knowledge, the diagnosis 'PAI of unknown etiology' does not automatically implicate gonadal insufficiency and might be neglected in the absence of a genetic diagnosis. Although gonadal function seems to be unaffected in our female patient so far, it appears to be impaired in her older brother. In other cases with homozygous Arg188Cys mutation, hypogonadism and infertility were reported. Close surveillance of gonadal function and discussion of possible preventive fertility measures (e.g. cryopreservation of sperms or oocytes) should be offered.

- Finding the appropriate dose of glucocorticoid and mineralocorticoid replacement therapy is often difficult, even in patients with PAI due to identical STAR mutations, e.g. Arg188Cys. Thus, genotype and phenotype do not correlate well. Individual dosing of drugs according to well-being of the patient seems essential. ACTH levels seem irrelevant as they tend to remain high despite adequate substitution with physiological or even supraphysiological doses of glucocorticoids.

\section{Case presentation}

A 26-year-old 46,XX female with childhood-onset PAI of unknown genetic cause was regularly seen at the outpatient clinic of endocrinology. Her two years older brother was diagnosed with PAI shortly after birth and started on treatment with glucocorticoids and mineralocorticoids. Initially, no signs of PAI were evident in the girl but at the age of 14 months, detailed investigations were performed due to progressive hyperpigmentation of the skin. Body weight was $9 \mathrm{~kg}$ (25th-50th percentile), height $72 \mathrm{~cm}$ (10th percentile) and head circumference $47 \mathrm{~cm}$ (75th-90th percentile). Laboratory analyses showed normal plasma sodium (143 nmol/L; ref. values: $136-145)$ and potassium ( $4.9 \mathrm{nmol} / \mathrm{L}$, ref. value: $3.5-5.0)$ levels, while chloride (106 nmol/L, 95-105) was just above normal. Bicarbonate was decreased (20.3 nmol/L, ref. values: $22-28$ ) and glucose normal $(4.2 \mathrm{nmol} / \mathrm{L}$, ref. values: 4.1-5.5). Hormonal analyses showed the constellation of subclinical PAI with elevated ACTH (>1500 ng/L, ref. values: 7.3-50.4), but normal cortisol ( $149 \mathrm{nmol} / \mathrm{L}$, ref. values: $55-690 \mathrm{nmol} / \mathrm{L})$. Renin activity was increased $(30.1 \mathrm{ng} / \mathrm{mL} / \mathrm{h}$, ref. values: $1.4-7.8)$ with normal aldosterone $(647 \mathrm{pmol} / \mathrm{L}$; ref. values: 180.3-2385). Testing for 21-hydroxylase antibodies was negative. Replacement therapy with $50 \mu \mathrm{g}$ fludrocortisone daily and $10 \mathrm{mg}$ hydrocortisone $\left(20 \mathrm{mg} / \mathrm{m}^{2} /\right.$ day) was initiated.

She was then regularly followed by the pediatric endocrinologist throughout her childhood without any reported abnormalities. At the age of 16 years, her height was $159 \mathrm{~cm}$ (10th-25th percentile) and weight $72 \mathrm{~kg}$ (90th-97th percentile). Blood pressure was 130/85 $\mathrm{mmHg}$. Secondary sex characteristics were fully developed and normal menstrual cycles were reported. At that age transition to adult endocrine care was performed. In the following years, doses of hydrocortisone were repeatedly adjusted because of elevated ACTH levels ( $>300 \mathrm{pmol} / \mathrm{L}$ ) and progressive hyperpigmentation of the skin on one hand and because of weight problems and to avoid Cushings on the other. Because of elevated blood pressure, fludrocortisone was temporarily stopped at the age of 19 years without prompting hypotension, orthostasis or hyperkalemia, but restarted two years later in order to save on hydrocortisone dose. 


\section{Investigation}

Current follow-up at the age of 26 years revealed no signs of cognitive or physical disabilities. She had a normal medical history concerning working and exercise capacity. Menstrual cycles were regular without hormonal contraception. Physical examination revealed a $1.60 \mathrm{~m}$ tall and slightly overweight (BMI: $28.1 \mathrm{~kg} / \mathrm{m}^{2}$ ) female patient. Blood pressure was $130 / 80 \mathrm{mmHg}$, pubertal development was adult, corresponding to Tanner stage V. Generalized hyperpigmentation of the skin was noted, predominately periorbital, in the hand lines, and the dorsal and plantar parts of the knees and joints. Under daily treatment with $25 \mathrm{mg}$ hydrocortisone and $50 \mu \mathrm{g}$ fludrocortisone, ACTH levels were increased (360 pmol/L; ref. values: $2.2-10.6)$. Renin levels were in normal range with $17.9 \mathrm{ng} / \mathrm{L}$ (ref. values: 2.6-27.2). Further laboratory work-up revealed normal electrolytes, normal HbA1c and a normal lipid profile. There were no signs of Cushings, especially no abdominal obesity.

\section{Outcome and follow-up}

Upon review of the case, consanguinity was noticed in the family and genetic testing for non-syndromic forms of PAI/FGD was recommended and performed after obtaining written informed consent.

PCR-based candidate gene testing was performed on genomic DNA extracted from blood leukocytes as described (5). Analyses for possible MC2R and MRAP mutations (FGD) were negative and a homozygous, single nucleotide change was found in the STAR gene (c.562C > T, p.Arg188Cys). The same homozygous mutation was also detected in her brother, while both parents were found to be heterozygous carriers of the mutation.

\section{Discussion}

Rare causes of PAI are linked to a growing number of genetic diseases and are either isolated adrenal disorders or part of a syndrome spectrum. Some of these genetic disorders are long known, while others have only been discovered recently. In a recently published large Turkish cohort, a genetic diagnosis was found in $81 \%$ (77/95) of children with PAI, in whom CAH, X-linked adrenoleukodystrophy, autoimmune triggered PAI, as well as known syndromic causes had been previously excluded (4). Different STAR mutations were detected in 11/95 children (6).

STAR is essential for the transport of cholesterol to the inner mitochondrial membrane of steroid producing organs (e.g. adrenal gland, ovaries and testes). More than
35 mutations are described so far. Clinical phenotype of patients with autosomal recessive STAR mutations varies considerably, butmaybegroupedinclassiccongenitallipoid adrenal hyperplasia (CLAH), where all steroidogenesis is disrupted, and non-classic CLAH (NCLAH), where typically only adrenal steroidogenesis is disrupted and thus an overlap with familial glucocorticoid deficiency (FGD) syndromes exists (7). CLAH is characterized by early and maybe life-threatening manifestation of adrenal insufficiency with fatal electrolyte shifts and a 46,XY disorder of sex development (DSD) in males as well as a lack of pubertal development in both sexes. By contrast, NCLAH manifests generally later as an adrenal deficiency phenotype only, although life-long follow-up of gonadal function is warranted. To date, 9 STAR mutations causing NCLAH have been reported. The Arg188Cys mutation (found in our patient) has been described in 7 families. Its in vitro activity assessed by pregnenolone production from cholesterol in cell models ranges from 8 to $14 \%$ compared to the wild-type STAR protein.

In our patient, glucocorticoid deficiency was confirmed repeatedly in lab tests, which is consistent with other cases harboring the Arg188Cys mutation (8). Mild mineralocorticoid deficiency was also demonstrated after fludrocortisone withdrawal, when plasma renin levels increased. Other patients with the Arg188Cys mutation showed inconsistently mineralocorticoid deficiency. Hence, the need for replacement of mineralocorticoids must be evaluated for every case individually, and may depend on salt intake (8).

In our female patient, sex steroid production does not seem to be affected so far; she reports regular menstrual cycles. In the literature, data on sex steroids and fertility in NCLAH are highly divergent. Therefore, regular evaluation of ovarian function and, if abnormal, timely hormonal replacement and possibility of oocyte preservation may be recommended.

The brother of our patient refused endocrine follow-up. He was last seen at the age of 18 years by the pediatric endocrinologist. Normal adult pubertal development was found. Interestingly, a handwritten note, which was found in the sister's record revealed possible male hypergonadotropic hypogonadism when he was in his early twenties. In other cases of homozygous Arg188Cys mutation, a broad range of impact on sex steroid synthesis and fertility is described. In boys and men, normal pubertal development and fatherhood as well as bilateral cryptorchidism and hypergonadotropic hypogonadism have been reported (9). Male fertility seems to be compromised by atrophy or lipid droplets in Leydig cells and reduced spermatogenesis (9). 
In women, regular menstrual cycles seem to be preserved, but successful pregnancy was not reported so far (8). The 'two-hit model' of LCAH suggests that Leydig cells in the testes are earlier damaged by lipid deposition because LH-driven steroidogenesis begins prenatally, continues after birth and is not silenced until puberty, as it is the case for steroidogenesis of the granulosa/theca cells in the ovaries (10)

Declaration of interest

The authors declare that there is no conflict of interest that could be perceived as prejudicing the impartiality of the research reported.

\section{Funding}

The work-up of this case was supported by Swiss National Science Foundation grant 320030-146127 to C E F and Instituto de Salud Carlos III (www.isciii.es/; Madrid, Spain) Centro de Investigación Biomédica en Red de Enfermedades Raras (CIBERER; http://www.ciberer.es/) U-712 to M F C and $L A$.

\section{Patient consent}

Written informed consent has been obtained from the patient.

\section{Author contribution statement}

Lukas Burget, Idea, clinical workup, manuscript writing; Laura Audí Parera, Genetic analyses; Monica Fernandez-Cancio, Genetic analyses; Rolf Graeni, Clinical work-up; Christoph Henzen, Clinical work-up, data interpretation, manuscript writing; Christa E Flück, Genetics, data analysis, manuscript design and writing, SNF grant holder and ethic approval for the study.

\section{References}

1 Flück CE. Update on pathogenesis of primary adrenal insufficiency: beyond steroid enzyme deficiency and autoimmune adrenal destruction. European Journal of Endocrinology 2017177 R99-R111. (https://doi.org/10.1530/EjE-17-0128)

2 Hsieh S \& White PC. Presentation of primary adrenal insufficiency in childhood. Journal of Clinical Endocrinology and Metabolism 201196 E925-E928. (https://doi.org/10.1210/jc.2011-0015)

3 Malikova J \& Flück CE. Novel insight into etiology, diagnosis and management of primary adrenal insufficiency. Hormone Research in Pediatrics 201482 145-157. (https://doi. org/10.1159/000363107)

4 Hahner S, Spinnler C, Fassnacht M, Burger-Stritt S, Lang K, Milovanovic D, Beuschlein F, Willenberg HS, Quinkler M \& Allolio B. High incidence of adrenal crisis in educated patients with chronic adrenal insufficiency: a prospective study. Journal of Clinical Endocrinology and Metabolism 2015100 407-416. (https://doi. org/10.1210/jc.2014-3191)

5 Flück CE, Pandey AV, Dick B, Camats N, Fernández-Cancio M, Clemente M, Gussinyé M, Carrascosa A, Mullis PE \& Audi L. Characterization of novel StAR (steroidogenic acute regulatory protein) mutations causing non-classic lipoid adrenal hyperplasia. PLOS ONE 20116 e20178. (https://doi.org/10.1371/journal. pone.0020178)

6 Guran T, Buonocore F, Saka N, Ozbek MN, Aycan Z, Bereket A, Bas F, Darcan S, Bideci A, Guven A, et al. Rare causes of primary adrenal insufficiency: genetic and clinical characterization of a Large Nationwide Cohort. Journal of Clinical Endocrinology and Metabolism 2016101 284-292. (https://doi.org/10.1210/jc.2015-3250)

7 Miller WL. Disorders in the initial steps of steroid hormone synthesis. Journal of Steroid Biochemistry and Molecular Biology 2017 165 18-37. (https://doi.org/10.1016/j.jsbmb.2016.03.009)

8 Baker BY, Lin L, Kim CJ, Raza J, Smith CP, Miller WL \& Achermann JC. Nonclassic congenital lipoid adrenal hyperplasia: a new disorder of the steroidogenic acute regulatory protein with very late presentation and normal male genitalia. Journal of Clinical Endocrinology and Metabolism 200691 4781-4785. (https://doi. org/10.1210/jc.2006-1565)

9 Metherell LA, Naville D, Halaby G, Begeot M, Huebner A, Nürnberg G, Nürnberg P, Green J, Tomlinson JW, Krone NP, et al. Nonclassic lipoid congenital adrenal hyperplasia masquerading as familial glucocorticoid deficiency. Journal of Clinical Endocrinology and Metabolism 200994 3865-3871. (https://doi.org/10.1210/jc.20090467)

10 Bose HS, Sugawara T, Strauss JF 3rd \& Miller WL. The pathophysiology and genetics of congenital lipoid adrenal hyperplasia. New England Journal of Medicine 1996335 1870-1878. (https://doi.org/10.1056/NEJM199612193352503) 